\title{
Perfect simulation of infinite range Gibbs measures and coupling with their finite range approximations
}

\author{
A. Galves \\ E. Löcherbach \\ E. Orlandi
}

September 28, 2009

\begin{abstract}
In this paper we address the questions of perfectly sampling a Gibbs measure with infinite range interactions and of perfectly sampling the measure together with its finite range approximations. We solve these questions by introducing a perfect simulation algorithm for the measure and for the coupled measures. The algorithm works for general Gibbsian interaction under requirements on the tails of the interaction. As a consequence we obtain an upper bound for the error we make when sampling from a finite range approximation instead of the true infinite range measure.
\end{abstract}

Key words : Coupling, perfect simulation, $\bar{d}$-distance, Gibbs measure, infinite range interactions, finite range approximations, interacting particle systems.

MSC 2000 : 82B20, 60K35, 60G60, 62M40.

\section{Introduction}

Consider a Gibbs measure with an infinite range potential and its finite range approximation obtained by truncating the interaction at a certain range. Can we construct a perfect sampling of the Gibbs measure with an infinite range potential? Further, if we make a local inspection of a perfect sampling of the finite range approximation, how often does it coincide with a sample from the original infinite range measure? We address these questions by introducing a new perfect simulation algorithm for the original Gibbs measure and a coupled perfect simulation algorithm for the original measure together with its finite range approximation.

By a perfect simulation algorithm we mean a function mapping a family of independent uniform random variables on the set of configurations having as law the infinite range Gibbs measure. By a coupled perfect simulation algorithm we mean a function mapping a family of independent uniform random variables on the set of pairs of configurations having as first marginal the original infinite range measure and as second marginal the finite range approximation. These functions are defined through two coupled Glauber dynamics having as reversible measures the original Gibbs measure and its finite range approximation, respectively. The perfect simulation algorithm produces coupled samples of the two measures.

The main difficulty in this constructive approach is that we have to deal with infinite range 
spin flip rates. This difficulty is overcome by a decomposition of the spin flip rates of the Glauber dynamics as a convex sum of local spin flip rates.

Our results are obtained under high temperature conditions and assuming that the interaction decays fast enough. We do not need any monotonicity property of the infinite range Gibbs measure. Under these conditions, we show that the algorithm that we propose stops almost surely, after a finite number of steps. Moreover, we give an upper bound, uniform in space, for the probability of local discrepancy between the coupled realizations of the two measures. This is the content of Theorem 1. As a corollary, Theorem 4 shows that the same upper bound holds for Ornstein's $\bar{d}$-distance between the two measures.

An upper bound for the $\bar{d}$-distance slightly worse than the one obtained in Theorem 4 can be obtained under less restrictive assumptions, by applying Dobrushin's contraction method. We refer the reader to the series of papers of Dobrushin (1968-1970) as well as to Presutti (2009) for a recent nice self-contained presentation of the subject. However, the contraction method is not constructive and does not provide an explicit sampling procedure for the measures.

The approach followed in the present paper was suggested by a recent work of Galves et al. (2008). There, the flip rates of an infinite range interacting multicolor system were decomposed as convex combinations of local range flip rates. With respect to this work, the novelty of what follows is that the decomposition is explicit and more suitable in view of the intended coupling result. The idea of decomposing flip rates can be traced back to Ferrari (1990) in which something with the same flavor was done for a finite range interacting particle system and to Ferrari et al. (2000) in which the probability transition of a stochastic chain of infinite order was decomposed as a convex combination of finite order probability transitions. We refer to Galves et al. (2008) for more details and related literature.

This paper is organized as follows. In Section 2, we present the basic notations and the main result, Theorem 1, In Section 3, we introduce the Glauber dynamics and the associated processes. The representation of the spin flip rates as convex combination of local rates is given in Theorem 2. In Section 4.1 we introduce the backward sketch process which is the basis of the perfect simulation algorithm and collect the main properties of this process in Proposition 3. Section 4.2 presents the coupled perfect simulation algorithm for the couple of measures. In Section 5 we prove Theorem 1 . We conclude the paper in Section 6 discussing how certain features of high temperature Gibbs systems can be obtained applying our construction. In particular we give results on Ornstein's $\bar{d}$-distance between the infinite range and the finite range Gibbs measure, and on the decay of correlations. These results are obtained under requirements stronger than the one needed using the Dobrushin contraction method, since they are based on our explicit coupled perfect simulation procedure.

Acknowledgments. The authors thank L. Bertini, P. Collet, P. Ferrari, R. Fernández, D. Gabrielli and E. Presutti for helpful discussions. We thank the Institut Henri Poincaré - Centre Emile Borel (workshop Mécanique Statistique, Probabilités et Systèmes de Particules 2008) for hospitality and support.

The authors have been partially supported by Prin07: 20078XYHYS (E.O.), by CNPq 305447/2008-4 (A.G.), ANR-08-BLAN-0220-01 (E.L.) and Galileo 2008-09 (E.L. and E.O.). This paper is part of PRONEX/FAPESP's project Stochastic behavior, critical phenomena 
and rhythmic pattern identification in natural languages (grant number 03/09930-9) and CNRS-FAPESP project Probabilistic phonology of rhythm. Ce travail a bénéficié d'une aide de l'Agence Nationale de la Recherche portant la référence ANR-08-BLAN-0220-01.

\section{Definitions and main results}

Let $A=\{-1,1\}$ and $S=A^{\mathbb{Z}^{d}}$ be the set of spin configurations. We endow $S$ with the product sigma algebra. We define on $\mathbb{Z}^{d}$ the $L^{1}$ norm, $\|i\|=\sum_{k=1}^{d}\left|i_{k}\right|$.

Configurations will be denoted by Greek letters $\sigma, \eta, \ldots$ A point $i \in \mathbb{Z}^{d}$ will be called a site. For any $i \in \mathbb{Z}^{d}$, notations $\sigma(i)$ and $\sigma_{i}$ will be used in order to denote the value of the configuration $\sigma$ at site $i$. By extension, for any subset $V \subset \mathbb{Z}^{d}, \sigma(V)$ and $\sigma_{V} \in A^{V}$ denote the restriction of the configuration $\sigma$ to the set of positions in $V$. If $V \subset \mathbb{Z}^{d}$ is a finite subset, we denote $V \Subset \mathbb{Z}^{d}$. In this case, we write $|V|$ for the cardinal of $V$. Finally, for any $i \in \mathbb{Z}^{d}$ and $\sigma \in S$, we shall denote $\sigma^{i}$ the modified configuration

$$
\sigma^{i}(j)=\sigma(j), \text { for all } j \neq i \text {, and } \sigma^{i}(i)=-\sigma(i) .
$$

Definition 1 An interaction is a collection $\left\{J_{B}, B \Subset \mathbb{Z}^{d}\right\}$ of real numbers indexed by finite subsets $B \Subset \mathbb{Z}^{d},|B| \geq 2$, which satisfies

$$
\sup _{i \in \mathbb{Z}^{d}} \sum_{B: i \in B}\left|J_{B}\right|<\infty
$$

For finite $B \subset \mathbb{Z}^{d}$ and $\sigma \in S$ let

$$
\chi_{B}(\sigma)=\prod_{i \in B} \sigma(i)
$$

Definition 2 A probability measure $\mu$ on $S$ is said to be a Gibbs measure relative to the interaction $\left\{J_{B}, B \Subset \mathbb{Z}^{d}\right\}$ if for all $i \in \mathbb{Z}^{d}$ and for any fixed $\zeta \in S$, a version of the conditional probability $\mu(\{\sigma: \sigma(i)=\zeta(i) \mid \sigma(j)=\zeta(j)$ for all $j \neq i\})$ is given by

$$
\mu(\{\sigma: \sigma(i)=\zeta(i) \mid \sigma(j)=\zeta(j) \text { for all } j \neq i\})=\frac{1}{1+\exp \left(-2 \sum_{B: i \in B} J_{B} \chi_{B}(\zeta)\right)} .
$$

To define the Gibbs measure $\mu^{[L]}$ relative to the interaction truncated at range $L$ we set

$$
B_{i}(L)=\left\{j \in \mathbb{Z}^{d}:\|j-i\| \leq L\right\} .
$$

Then $\mu^{[L]}$ is the probability measure on $S$ such that for all $i \in \mathbb{Z}^{d}$ and for any fixed $\zeta \in S$, a version of the conditional probability $\mu^{[L]}(\{\sigma: \sigma(i)=\zeta(i) \mid \sigma(j)=\zeta(j)$ for all $j \neq i\})$ is given by

$$
\begin{aligned}
\mu^{[L]}(\{\sigma: \sigma(i)=\zeta(i) \mid \sigma(j)=\zeta(j) \text { for all } j \neq i\}) & \\
& =\frac{1}{1+\exp \left(-2 \sum_{B: i \in B, B \subset B_{i}(L)} J_{B} \chi_{B}(\zeta)\right)} .
\end{aligned}
$$

We consider the interaction $J_{B}^{\beta}=\beta J_{B}$ where $\beta$ is a positive parameter. The associated Gibbs measures will be denoted by $\mu$ and $\mu^{[L]}$, omitting to write the explicit dependence 
on $\beta$. We denote by $\left(X(i), i \in \mathbb{Z}^{d}\right),\left(X^{[L]}(i), i \in \mathbb{Z}^{d}\right)$ two random configurations on $S$ distributed according to $\mu$ and $\mu^{[L]}$, respectively.

All processes that we consider in this paper will be constructed as functions of an independent family of uniform random variables $\left(U_{n}(i), n \in \mathbb{N}, i \in \mathbb{Z}^{d}\right)$. Denote $(\Omega, \mathcal{A}, P)$ the probability space on which is defined the independent family of uniform random variables.

Definition 3 A perfect sampling algorithm of the measure $\mu$ is a map $F$ from $[0,1]^{\mathbb{N} \times \mathbb{Z}^{d}}$ to $\{-1,+1\}^{\mathbb{Z}^{d}}$, such that

$$
F\left(U_{n}(j), n \in \mathbb{N}, j \in \mathbb{Z}^{d}\right) \text { has distribution } \mu .
$$

A coupled perfect sampling algorithm for the pair $\left(\left(X(i), X^{[L]}(i)\right), i \in \mathbb{Z}^{d}\right)$ is a map $G$ from $[0,1]^{\mathbb{N} \times \mathbb{Z}^{d}}$ to $\{-1,+1\}^{\mathbb{Z}^{d} \times \mathbb{Z}^{d}}$, such that

$$
G\left(U_{n}(j), n \in \mathbb{N}, j \in \mathbb{Z}^{d}\right) \text { has marginals }\left(X(i), i \in \mathbb{Z}^{d}\right) \text { and }\left(X^{[L]}(i), i \in \mathbb{Z}^{d}\right) \text {. }
$$

Since $F$ produces an unbiased sample from the target distribution $\mu$, the algorithm is denoted "perfect".

Definition 4 The perfect sampling algorithm $F$ of the measure $\mu$ is said to stop almost surely after a finite number of steps if for every site $i \in \mathbb{Z}^{d}$, there exists a finite subset $F^{(i)}$ of $\mathbb{Z}^{d}$ and a finite random variable $N_{S T O P}^{(i)} \geq 1$ such that if

$$
U_{n}^{\prime}(j)=U_{n}(j) \text { for all } j \in F^{(i)}, 1 \leq n \leq N_{S T O P}^{(i)}
$$

then

$$
F\left(U_{n}(j), n \in \mathbb{N}, j \in \mathbb{Z}^{d}\right)(i)=F\left(U_{n}^{\prime}(j), n \in \mathbb{N}, j \in \mathbb{Z}^{d}\right)(i) .
$$

Note that (2.5) implies that $F\left(U_{n}(j), n \in \mathbb{N}, j \in \mathbb{Z}^{d}\right)(i)$ depends only on $U_{n}(j)$ for $1 \leq n \leq$ $N_{S T O P}^{(i)}$. The following theorem is our main result.

Theorem 1 Assume that

$$
\sup _{i \in \mathbb{Z}^{d}} \sum_{k}\left|B_{i}(k)\right|\left(\sum_{B: i \in B, B \subset B_{i}(k), B \not \subset B_{i}(k-1)}\left|J_{B}\right|\right)<\infty .
$$

1. There exists $\beta_{c}>0$ such that for any $\beta<\beta_{c}$ there is a perfect sampling algorithm of $\mu$. This algorithm stops after a finite number of steps almost surely and

$$
\sup _{i \in \mathbb{Z}^{d}} E\left(N_{S T O P}^{(i)}\right) \leq \frac{1}{\gamma}
$$

where $\gamma$ is given in (4.37).

2. Moreover, for any $\beta<\beta_{c}$, there exists a coupled perfect sampling algorithm for the pair $\left(\left(X(i), X^{[L]}(i)\right), i \in \mathbb{Z}^{d}\right)$ satisfying

$$
\sup _{i \in \mathbb{Z}^{d}} P\left(X(i) \neq X^{[L]}(i)\right) \leq \frac{1}{\gamma} \sup _{i \in \mathbb{Z}^{d}}\left(1-e^{-\beta \sum_{B: i \in B, B \not \subset B_{i}(L)}\left|J_{B}\right|}\right) .
$$


Remark 1 The constant $\beta_{c}$ is the solution of equation (5.39) in Section 5 .

Remark 2 In case of an interaction defined through a pairwise potential $\{J(i, j),(i, j) \in$ $\left.\mathbb{Z}^{d} \times \mathbb{Z}^{d}\right\}$ with $J(i, i)=0$ for $i \in \mathbb{Z}^{d}$, condition (2.6) of Theorem 1 reads as

$$
\sup _{i \in \mathbb{Z}^{d}} \sum_{k}\left|B_{i}(k)\right|\left(\sum_{j:\|i-j\|=k}|J(i, j)|\right)<\infty .
$$

In this case,

$$
\sup _{i \in \mathbb{Z}^{d}} P\left(X(i) \neq X^{[L]}(i)\right) \leq \frac{1}{\gamma} \sup _{i \in \mathbb{Z}^{d}}\left(1-e^{-\beta \sum_{j:\|i-j\|>L}|J(i, j)|}\right) .
$$

The above perfect sampling algorithms are based on a coupled construction of two processes having $\mu$ and $\mu^{[L]}$ as reversible measures. These two processes will be introduced in the next section. The definition of the algorithms can only be given in Section 4. The proof of Theorem 1 is given in Section 5 .

\section{Glauber dynamics}

We now introduce a Glauber dynamics having $\mu$ as reversible measure. This is an interacting particle system $\left(\sigma_{t}(i), i \in \mathbb{Z}^{d}, t \in \mathbb{R}\right)$ taking values in $S$. Sometimes we will also use the short notation $\left(\sigma_{t}\right)_{t}$ for the interacting particle system. To describe the process, we need some extra notation.

For any $i \in \mathbb{Z}^{d}, \sigma \in\{-1,1\}^{\mathbb{Z}^{d}}$, and $\beta>0$, we define $c_{i}(\sigma)$, which is the rate at which the spin $i$ flips when the system is in the configuration $\sigma$, as

$$
c_{i}(\sigma)=\exp \left(-\beta \sum_{B: i \in B} J_{B} \chi_{B}(\sigma)\right) .
$$

The generator $\mathcal{G}$ of the process $\left(\sigma_{t}\right)_{t}$ is defined on cylinder functions $f: S \rightarrow \mathbb{R}$ as follows

$$
\mathcal{G} f(\sigma)=\sum_{i \in \mathbb{Z}^{d}} c_{i}(\sigma)\left[f\left(\sigma^{i}\right)-f(\sigma)\right]
$$

Observe that the condition (2.6) of Theorem 1 implies that

$$
\sup _{i} \sum_{B: i \in B}|B|\left|J_{B}\right|<\infty
$$

where $|B|$ designs the cardinal of the set $B$. Hence the rates $c_{i}(\sigma)$ are uniformly bounded in $i$ and $\sigma$, and

$$
\sup _{i \in \mathbb{Z}^{d}} \sum_{j \in \mathbb{Z}^{d}} \sup _{\sigma}\left|c_{i}(\sigma)-c_{i}\left(\sigma^{j}\right)\right|<\infty .
$$

Therefore, Theorem 3.9 of Chapter 1 of Liggett (1985) implies that $\mathcal{G}$ is the generator of a Markov process $\left(\sigma_{t}\right)_{t}$ on $S S$. By construction, the process $\left(\sigma_{t}\right)_{t}$ is reversible with respect to the Gibbs measure $\mu$ corresponding to the interaction $J_{B}^{\beta}=\beta J_{B}$. 
For any $\ell \geq 1$, we now define a Glauber dynamics having $\mu^{[\ell]}$ as reversible measure. More precisely, we consider the Markov process $\left(\sigma_{t}^{[\ell]}\right)_{t}$ on $S S$ having generator

$$
\mathcal{G}^{[\ell]} f(\sigma)=\sum_{i \in \mathbb{Z}^{d}} c_{i}^{[\ell]}(\sigma)\left[f\left(\sigma^{i}\right)-f(\sigma)\right]
$$

where the rates $c_{i}^{[\ell]}$ are given by

$$
c_{i}^{[\ell]}(\sigma)=r_{i}^{[\ell]} \exp \left(-\beta \sum_{B: i \in B, B \subset B_{i}(\ell)} J_{B} \chi_{B}(\sigma)\right)
$$

and

$$
r_{i}^{[\ell]}=e^{-\beta \sum_{B: i \in B, B \not \subset B_{i}(\ell)}\left|J_{B}\right|} .
$$

Let us stress the fact that compared to the usual definition of a Glauber dynamics, an extra factor $r_{i}^{[\ell]}$ appears in the definition of the rates $c_{i}^{[\ell]}$. This extra factor is important in view of the intended coupling of both processes $\sigma_{t}$ and $\sigma_{t}^{[\ell]}$ (see Theorem 2 below) and does not change the equilibrium behavior of the process as is shown in the next proposition.

Proposition 1 The process $\left(\sigma_{t}^{[\ell]}\right)_{t}$ is reversible with respect to the Gibbs measure $\mu^{[\ell]}$ relative to the pairwise interaction $J_{\beta}$ truncated at range $\ell$.

Proof By construction, we have that

$$
\frac{c_{i}^{[\ell]}(\sigma)}{c_{i}^{[\ell]}\left(\sigma^{i}\right)}=\frac{\mu^{[\ell]}\left(\sigma^{i}\right)}{\mu^{[\ell]}(\sigma)}
$$

Thus, by Proposition 2.7 of Chapter IV of Liggett (1985), it follows that $\left(\sigma_{t}^{[\ell]}\right)_{t}$ is reversible with respect to $\mu^{[\ell]}$.

The main tool to prove Theorem 1 is a coupled construction of the processes $\left(\sigma_{t}(i), i \in\right.$ $\left.\mathbb{Z}^{d}, t \in \mathbb{R}\right)$ and $\left(\sigma_{t}^{[\ell]}(i), i \in \mathbb{Z}^{d}, t \in \mathbb{R}\right)$ for $\ell=L$, using the basic family of uniform random variables $\left(U_{n}(i), n \in \mathbb{N}, i \in \mathbb{Z}^{d}\right)$. This coupling is based on a decomposition of the spin flip rates $c_{i}(\sigma)$ as a convex combination of local range spin flip rates.

To present this decomposition we need the following notation. We define

$$
M_{i}=2 e^{\beta \sum_{B: i \in B}\left|J_{B}\right|},
$$

and the sequence $\left(\lambda_{i}(k)\right)_{k \geq 0}$ as follows

$$
\lambda_{i}(k)= \begin{cases}e^{-2 \beta \sum_{B: i \in B}\left|J_{B}\right|} & \text { if } k=0, \\ e^{-\beta \sum_{B: i \in B, B \not \subset B_{i}(1)}\left|J_{B}\right|}-e^{-2 \beta \sum_{B: i \in B}\left|J_{B}\right|} & \text { if } k=1, \\ e^{-\beta \sum_{B: i \in B, B \not \subset B_{i}(k)}\left|J_{B}\right|}-e^{-\beta \sum_{B: i \in B, B \not \subset B_{i}(k-1)}\left|J_{B}\right|} & \text { if } k>1 .\end{cases}
$$

Finally, for all $\sigma \in S$ and for any $k \geq 2$, we define the update probabilities

$$
\begin{aligned}
p_{i}^{[k]}(-\sigma(i) \mid \sigma)= & \frac{1}{M_{i}} e^{-\beta \sum_{B: i \in B, B \subset B_{i}(k-1)} J_{B} \chi_{B}(\sigma)} \\
& \frac{e^{-\beta \sum_{B: i \in B, B \subset B_{i}(k), B \not \subset B_{i}(k-1)} J_{B} \chi_{B}(\sigma)}-e^{-\beta \sum_{B: i \in B, B \subset B_{i}(k), B \not \subset B_{i}(k-1)}\left|J_{B}\right|}}{1-e^{-\beta \sum_{B: i \in B, B \subset B_{i}(k), B \not \subset B_{i}(k-1)}\left|J_{B}\right|}},
\end{aligned}
$$


and for $k=1$,

$$
p_{i}^{[1]}(-\sigma(i) \mid \sigma)=\frac{1}{M_{i}} \frac{e^{-\beta \sum_{B: i \in B, B \subset B_{i}(1)} J_{B} \chi_{B}(\sigma)}-e^{-\beta \sum_{B: i \in B, B \subset B_{i}(1)}\left|J_{B}\right|}}{1-e^{-2 \beta \sum_{B: i \in B, B \subset B_{i}(1)}\left|J_{B}\right|} e^{-\beta \sum_{B: i \in B, B \not \subset B_{i}(1)}\left|J_{B}\right|}} .
$$

To obtain a probability measure on $A$, we define for all $k \geq 1$,

$$
p_{i}^{[k]}(\sigma(i) \mid \sigma)=1-p_{i}^{[k]}(-\sigma(i) \mid \sigma)
$$

Finally, for $k=0$, we define

$$
p_{i}^{[0]}(1)=p_{i}^{[0]}(-1)=\frac{1}{2} .
$$

Since this last probability does not depend on the site $i$ we omit the subscript $i$. Note that, by construction, for any integer $k \geq 1$, the probabilities $p_{i}^{[k]}(a \mid \sigma)$ depend only on $\sigma\left(B_{i}(k)\right)$.

Now we state the decomposition theorem.

Theorem 2 Let us assume that the uniform summability condition (2.1) holds.

1. The sequence $\left(\lambda_{i}(k)\right)_{k \geq 0}$ defines a probability distribution on the set of positive integers $\{0,1, \ldots\}$.

2. For any $\ell \geq 1, \sigma \in S$, the following decomposition holds

$$
c_{i}^{[\ell]}(\sigma)=M_{i}\left[\lambda_{i}(0) \frac{1}{2}+\sum_{k=1}^{\ell} \lambda_{i}(k) p_{i}^{[k]}(-\sigma(i) \mid \sigma)\right] .
$$

3. For any $\sigma \in S$, the following decomposition holds

$$
c_{i}(\sigma)=M_{i}\left[\lambda_{i}(0) \frac{1}{2}+\sum_{k=1}^{\infty} \lambda_{i}(k) p_{i}^{[k]}(-\sigma(i) \mid \sigma)\right] .
$$

Proof Item 1 follows directly from the definition and (2.1).

To prove item 2 , set

$$
c_{i}^{[0]}(\sigma)=\frac{1}{2} M_{i} \lambda_{i}(0)
$$

and observe that

$$
c_{i}^{[\ell]}(\sigma)=\sum_{k=1}^{\ell}\left[c_{i}^{[k]}(\sigma)-c_{i}^{[k-1]}(\sigma)\right]+c_{i}^{[0]}(\sigma),
$$

where the differences $c_{i}^{[k]}(\sigma)-c_{i}^{[k-1]}(\sigma)$ are always positive. Since by definition

$$
c_{i}^{[k]}(\sigma)-c_{i}^{[k-1]}(\sigma)=M_{i} \lambda_{i}(k) p_{i}^{[k]}(-\sigma(i) \mid \sigma),
$$

we obtain the decomposition stated in item 2 .

To prove item 3, observe that the uniform summability condition (2.1) implies that

$$
\lim _{k \rightarrow \infty} c_{i}^{[k]}(\sigma)=c_{i}(\sigma)
$$


since $r_{i}^{[k]} \rightarrow 0$ for $k \rightarrow \infty$. Thus, taking into account item 2 , we obtain the desired decomposition.

To present the coupling, it is convenient to rewrite the generator of the Glauber dynamics given in (3.10) as

$$
\mathcal{G} f(\sigma)=\sum_{i \in \mathbb{Z}^{d}} \sum_{a \in A} c_{i}(a \mid \sigma)\left[f\left(\sigma^{i, a}\right)-f(\sigma)\right]
$$

where

$$
\sigma^{i, a}(j)=\sigma(j), \text { for all } j \neq i \text {, and } \sigma^{i, a}(i)=a,
$$

and where

$$
c_{i}(a \mid \sigma)= \begin{cases}c_{i}(\sigma) & \text { if } a=-\sigma(i) \\ M_{i}-c_{i}(\sigma) & \text { if } a=\sigma(i)\end{cases}
$$

In a similar way, for any integer $\ell \geq 1$, we define the rates

$$
c_{i}^{[\ell]}(a \mid \sigma)= \begin{cases}c_{i}^{[\ell]}(\sigma) & \text { if } a=-\sigma(i), \\ M_{i} \sum_{k=0}^{\ell} \lambda_{i}(k)-c_{i}^{[\ell]}(\sigma) & \text { if } a=\sigma(i)\end{cases}
$$

and the generator

$$
\mathcal{G}^{[\ell]} f(\sigma)=\sum_{i \in \mathbb{Z}^{d}} \sum_{a \in A} c_{i}^{[\ell]}(a \mid \sigma)\left[f\left(\sigma^{i, a}\right)-f(\sigma)\right]
$$

This amounts to include in the generators the rates of invisible jumps in which the spin $i$ is updated with the same value it had before. Obviously the generator defined in (3.10) (and in (3.12)) defines the same stochastic dynamics as the generator given in (3.18) (and in (3.21), respectively).

With this representation, we have the following corollary of Theorem 2 ,

\section{Corollary 1}

$$
\mathcal{G} f(\sigma)=\sum_{i \in \mathbb{Z}^{d}} \sum_{a \in A} \sum_{k \geq 0} M_{i} \lambda_{i}(k) p_{i}^{[k]}(a \mid \sigma)\left[f\left(\sigma^{i, a}\right)-f(\sigma)\right]
$$

and for any $\ell \geq 1$,

$$
\mathcal{G}^{[\ell]} f(\sigma)=\sum_{i \in \mathbb{Z}^{d}} \sum_{a \in A} \sum_{k=0}^{\ell} M_{i} \lambda_{i}(k) p_{i}^{[k]}(a \mid \sigma)\left[f\left(\sigma^{i, a}\right)-f(\sigma)\right] .
$$

From now on, we fix $\ell=L$. The decomposition given in Corollary 1 suggests the following construction of the Glauber dynamics having $\mathcal{G}$ and $\mathcal{G}^{[L]}$ as infinitesimal generator. For each site $i \in \mathbb{Z}^{d}$ consider a Poisson point process $N^{i}$ having rate $M_{i}$. The Poisson processes corresponding to distinct sites are all independent. If, at time $t$, the Poisson clock associated to site $i$ rings, we choose a range $k$ with probability $\lambda_{i}(k)$ independently of everything else. Then we update the value of the configuration at this site by choosing a symbol $a$ with probability $p_{i}^{[k]}\left(a \mid \sigma\left(B_{i}(k)\right)\right)$ depending only on the configurations inside the set $B_{i}(k)$. It is clear that using this decomposition, we can construct both processes $\sigma_{t}$ and $\sigma_{t}^{[L]}$ in a coupled way, starting from any initial configuration. 
Actually we can do better than this. We can make a perfect simulation of the pair of measures $\mu$ and $\mu^{[L]}$ which are the invariant probability measures of the processes having generators $\mathcal{G}$ and $\mathcal{G}^{[L]}$ respectively.

Let us explain how we sample the configuration at a fixed site $i \in \mathbb{Z}^{d}$ under the measure $\mu$. The simulation procedure has two stages. In the first stage we determine the set of sites whose spins influence the spin at site $i$ under equilibrium. We call this stage backward sketch procedure. It is done by climbing up from time 0 back to the past a reverse time Poisson point process with rate $M_{i}$ until the last time the Poisson clock rung. At that time, choose a range $k$ with probability $\lambda_{i}(k)$. If $k=0$, we decide the value of the spin with probability $\frac{1}{2}$ independently of anything else. If $k$ is different from zero, we restart the above procedure from any of the sites $j \in B_{i}(k)$. The procedure stops whenever each site involved in this backward time evolution has chosen a range 0.

When this occurs, we can start the second stage, in which we go back to the future assigning spins to all sites visited during the first stage. We call this procedure forward spin assignment procedure. This is done from the past to the future by using the update probabilities $p_{i}^{[k]}$ starting at the sites which ended the first procedure by choosing range 0 . For each one of these sites a spin is chosen by tossing a fair coin. The values obtained in this way enter successively in the choice of the values of the spins depending on a neighborhood of range greater or equal to 1.

In the case of the measure $\mu^{[L]}$ we use the same procedure, but only considering the choices of $k$ which are smaller or equal to $L$. These procedures will be described formally in the next section.

\section{Perfect simulation of the measure $\mu$ and coupling}

\subsection{Backward sketch procedure}

Before describing formally the two algorithms described at the end of the last section, let us define the stochastic process which is behind the backward sketch procedure. Our aim is to define, for every site $i \in \mathbb{Z}^{d}$, a process $\left(C_{s}^{(i)}\right)_{s \geq 0}$ taking values in the set of finite subsets of $\mathbb{Z}^{d}$, such that $C_{s}^{(i)}$ is the set of sites at time $-s$ whose spins affect the spin of site $i$ at time $t=0$. This is done as follows.

For each $i \in \mathbb{Z}^{d}$, denote by $\ldots T_{-2}^{i}<T_{-1}^{i}<T_{0}^{i}<0<T_{1}^{i}<T_{2}^{i}<\ldots$ the occurrence times of the rate $M_{i}$ Poisson point process $N^{i}$ on the real line. The Poisson point processes associated to different sites are independent. To each point $T_{n}^{i}$ associate an independent mark $K_{n}^{i}$ according to the probability distribution $\left(\lambda_{i}(k)\right)_{k \geq 0}$. As usual, we identify the Poisson point processes and the counting measures through the formula

$$
N^{i}[s, t]=\sum_{n \in \mathbb{Z}} \mathbf{1}_{\left\{s \leq T_{n}^{i} \leq t\right\}} .
$$

The backward point process starting at time 0 , associated to site $i \in \mathbb{Z}^{d}$ is defined as

$$
T_{n}^{(i, 0)}=-T_{-n+1}^{i}, \text { for any } n \geq 1 .
$$

We also define the associated marks

$$
K_{n}^{(i, 0)}=K_{-n+1}^{i}
$$


and for any $k \geq 0$, the backward $k$-marked Poisson point process starting at time 0 as

$$
N^{(i, k)}[s, u]=\sum_{n \in \mathbb{Z}} \mathbf{1}_{\left\{s \leq T_{n}^{(i, 0)} \leq u\right\}} \mathbf{1}_{\left\{K_{n}^{(i, 0)}=k\right\}} \cdot
$$

To define the backward sketch process we need to introduce a family of transformations $\left\{\pi^{(i, k)}, i \in \mathbb{Z}^{d}, k \geq 0\right\}$ on $\mathcal{F}\left(\mathbb{Z}^{d}\right)$, the set of finite subsets of $\mathbb{Z}^{d}$, defined as follows. For any unitary set $\{j\}$ and $k \geq 1$,

$$
\pi^{(i, k)}(\{j\})= \begin{cases}B_{i}(k), & \text { if } j=i \\ \{j\}, & \text { otherwise. }\end{cases}
$$

For $k=0$, we define

$$
\pi^{(i, 0)}(\{j\})= \begin{cases}\emptyset, & \text { if } j=i, \\ \{j\}, & \text { otherwise. }\end{cases}
$$

For any set finite set $F \subset \mathbb{Z}^{d}$, we define similarly

$$
\pi^{(i, k)}(F)=\cup_{j \in F} \pi^{(i, k)}(\{j\}) .
$$

The backward sketch process starting at site $i$ at time 0 will be denoted by $\left(C_{s}^{(i)}\right)_{s \geq 0}$. The set $C_{s}^{(i)}$ is the set of sites at time $-s$ whose spins affect the spin of site $i$ at time $t=0$. The evolution of this process is defined through the following equation. $C_{0}^{(i)}=\{i\}$, and

$$
f\left(C_{s}^{(i)}\right)=f\left(C_{0}^{(i)}\right)+\sum_{k \geq 0} \sum_{j \in \mathbb{Z}^{d}} \int_{0}^{s}\left[f\left(\pi^{(j, k)}\left(C_{u-}^{(i)}\right)\right)-f\left(C_{u-}^{(i)}\right)\right] N^{(j, k)}(d u),
$$

where $f: \mathcal{F}\left(\mathbb{Z}^{d}\right) \rightarrow \mathbb{R}$ is any bounded cylinder function. This family of equations characterizes completely the time evolution $\left(C_{s}^{(i)}\right)_{s \geq 0}$.

In a similar way, for the truncated process, we define its associated backward sketch process by

$$
f\left(C_{s}^{[L],(i)}\right)=f\left(C_{0}^{[L],(i)}\right)+\sum_{k=0}^{L} \sum_{j \in \mathbb{Z}^{d}} \int_{0}^{s}\left[f\left(\pi^{(j, k)}\left(C_{u-}^{[L],(i)}\right)\right)-f\left(C_{u-}^{[L],(i)}\right)\right] N^{(j, k)}(d u),
$$

where we use the same Poisson point processes $N^{(j, k)}, 0 \leq k \leq L$, as in (4.28).

The following proposition summarizes the properties of the family of processes defined above.

Proposition 2 For any site $i \in \mathbb{Z}^{d}, C_{s}^{(i)}$ and $C_{s}^{[L],(i)}, s \in \mathbb{R}^{+}$, are Markov jump processes having as infinitesimal generator,

$$
\mathcal{L} f(C)=\sum_{i \in C} \sum_{k \geq 1} M_{i} \lambda_{i}(k)\left[f\left(C \cup B_{i}(k)\right)-f(C)\right]+\lambda_{i}(0)[f(C \backslash\{i\})-f(C)],
$$

with initial condition at time $t=0, C_{0}^{(i)}=\{i\}$, and

$$
\mathcal{L}^{[L]} f(C)=\sum_{i \in C} \sum_{k=1}^{L} M_{i} \lambda_{i}(k)\left[f\left(C \cup B_{i}(k)\right)-f(C)\right]+\lambda_{i}(0)[f(C \backslash\{i\})-f(C)],
$$

with initial condition at time $t=0, C_{0}^{[L],(i)}=\{i\}$, respectively. Here $f: \mathcal{F}\left(\mathbb{Z}^{d}\right) \rightarrow \mathbb{R}$ is any bounded cylindrical function. 
Let

$$
T_{S T O P}^{(i)}=\inf \left\{s: C_{s}^{(i)}=\emptyset\right\}
$$

be the relaxation time. We introduce the sequence of successive jump times $\tilde{T}_{n}^{(i)}, n \geq 1$, of processes $N^{(j, k)}$ whose jumps occur in (4.28). Let $\tilde{T}_{1}^{(i)}=T_{1}^{(i, 0)}$ and define successively for $n \geq 2$

$$
\left.\left.\tilde{T}_{n}^{(i)}=\inf \left\{t>\tilde{T}_{n-1}^{(i)}: \exists j \in C_{\tilde{T}_{n-1}^{(i)}}^{(i)}, \exists k: N^{(j, k)}(] \tilde{T}_{n-1}^{(i)}, t\right]\right)=1\right\} .
$$

Now we put

$$
\mathbf{C}_{n}^{(i)}=C_{\tilde{T}_{n}^{(i)}}^{(i)}
$$

Finally, notice that $N_{S T O P}^{(i)}$ of Definition 4 is equal to

$$
N_{S T O P}^{(i)}=\inf \left\{n: \mathbf{C}_{n}^{(i)}=\emptyset\right\}
$$

This is the number of steps of the backward sketch process. For the algorithm to be successful, it is crucial to show that both relaxation time $T_{S T O P}^{(i)}$ and the number of steps $N_{S T O P}^{(i)}$ are finite. This is the content of next theorem.

Proposition 3 Under the requirement

$$
\sup _{i \in \mathbb{Z}^{d}} \sum_{k \geq 1}\left|B_{i}(k)\right| \lambda_{i}(k)<1
$$

we have uniformly for any $i \in \mathbb{Z}^{d}$,

$$
P\left(T_{S T O P}^{(i)}>t\right) \leq e^{-\gamma t}
$$

and

$$
E\left(N_{S T O P}^{(i)}\right) \leq \frac{1}{\gamma}
$$

where

$$
\gamma=1-\sup _{i \in \mathbb{Z}^{d}} \sum_{k \geq 1}\left|B_{i}(k)\right| \lambda_{i}(k)
$$

The proof of Proposition 3 will be given at the end of Section 5 below.

\subsection{Algorithms}

We now go to the crucial point of precisely defining the maps $F$ and $G$ whose existence is claimed in Theorem 1. These maps are implicitly defined by the backward sketch procedure and the forward spin assignment procedure. Let us give the algorithmic like description of these procedures. The following variables will be used.

- $N$ is an auxiliary variables taking values in the set of non-negative integers $\{0,1,2, \ldots\}$

- $N_{S T O P}^{(i)}$ is a counter taking values in the set of non-negative integers $\{0,1,2, \ldots\}$

- $N_{S T O P}^{[L],(i)}$ is a counter taking values in the set of non-negative integers $\{0,1,2, \ldots\}$ 
- $I$ is a variable taking values in $\mathbb{Z}^{d}$

- $I^{[L]}$ is a variable taking values in $\mathbb{Z}^{d}$

- $K$ is a variable taking values in $\{0,1, \ldots\}$

- $K^{[L]}$ is a variable taking values in $\{0,1, \ldots\}$

- $B$ is an array of elements of $\mathbb{Z}^{d} \times\{0,1, \ldots\}$

- $B^{[L]}$ is an array of elements of $\mathbb{Z}^{d} \times\{0,1, \ldots\}$

- $C$ is a variable taking values in the set of finite subsets of $\mathbb{Z}^{d}$

- $C^{[L]}$ is a variable taking values in the set of finite subsets of $\mathbb{Z}^{d}$

- $W$ is an auxiliary variable taking values in $A$

- $X$ is a function from $\mathbb{Z}^{d}$ to $A \cup\{\Delta\}$, where $\Delta$ is some extra symbol that does not belong to $A$

- $X^{[L]}$ is a function from $\mathbb{Z}^{d}$ to $A \cup\{\Delta\}$, where $\Delta$ is some extra symbol that does not belong to $A$

We will present the backward sketch procedure only for $G$, the coupled perfect simulation algorithm. The backward sketch procedure for $F$ can be immediately deduced ignoring the steps from 11. to 18. concerning the variables with superscript $L$.

Algorithm 1 Backward sketch procedure

1. Input: $\{i\} ;$ Output: $N_{S T O P}^{(i)}, N_{S T O P}^{[L],(i)}, B, B^{[L]}$

2. $N \leftarrow 0, N_{S T O P}^{(i)} \leftarrow 0, N_{S T O P}^{[L],(i)} \leftarrow 0, B \leftarrow \emptyset, B^{[L]} \leftarrow \emptyset, C \leftarrow\{i\}, C^{[L]} \leftarrow\{i\}$

3. WHILE $C \neq \emptyset$

4. $N \leftarrow N+1$

5. Choose independent random variables $S_{N}^{(j, k)}$ having exponential distribution of parameter $M_{j} \lambda_{j}(k)$ for any $j \in C$ and for any $k \geq 0$

6. Choose $(I, K)=\arg \min \left\{S_{N}^{(j, k)}, j \in C, k \geq 0\right\}$

7. IF $K=0, C \leftarrow C \backslash\{I\}$

8. ELSE $C \leftarrow C \cup B_{I}(K)$

9. ENDIF

10. $B(N) \leftarrow(I, K)$

11. IF $C^{[L]} \neq \emptyset$

12. $N_{S T O P}^{[L],(i)} \leftarrow N$ 
13. Choose $\left(I^{[L]}, K^{[L]}\right)=\arg \min \left\{S_{N}^{(j, k)}, j \in C, 0 \leq k \leq L\right\}$

14. $\quad$ IF $K^{[L]}=0, C^{[L]} \leftarrow C^{[L]} \backslash\left\{I^{[L]}\right\}$

15. $\quad$ ELSE $C^{[L]} \leftarrow C^{[L]} \cup B_{I^{[L]}}\left(K^{[L]}\right)$

16. ENDIF

17. $\quad B^{[L]}(N) \leftarrow\left(I^{[L]}, K^{[L]}\right)$

18. ENDIF

19. ENDWHILE

20. $N_{S T O P}^{(i)} \leftarrow N$

21. RETURN $N_{S T O P}^{(i)}, N_{S T O P}^{[L],(i)}, B, B^{[L]}$

If $B=B^{[L]}$, then we use the following Forward spin assignment procedure to sample $X(i)=X^{[L]}(i)$. This is the algorithmic translation of the ideas presented in the last paragraph of Section 4 . If $B \neq B^{[L]}$, then we use the Forward spin assignment procedure twice independently, starting with input $N_{S T O P}^{(i)}, B$ in order to sample $X(i)$ and starting with input $N_{S T O P}^{[L],(i)}, B^{[L]}$ in order to sample $X^{[L]}(i)$.

$\underline{\text { Algorithm } 2 \text { Forward spin assignment procedure }}$

1. Input: $N_{S T O P}^{(i)}, B ;$ Output: $\{X(i)\}$

2. $N \leftarrow N_{S T O P}^{(i)}$

3. $X(j) \leftarrow \Delta$ for all $j \in \mathbb{Z}^{d}$

4. WHILE $N \geq 1$

5. $(I, K) \leftarrow B(N)$.

6. IF $K=0$ choose $W$ randomly in $A$ according to the probability distribution

$$
P(W=v)=p_{I}^{[0]}(v)=\frac{1}{2}
$$

7. ELSE choose $W$ randomly in $A$ according to the probability distribution

$$
P(W=v)=p_{I}^{[K]}(v \mid X)
$$
8. ENDIF
9. $X(I) \leftarrow W$
10. $N \leftarrow N-1$
11. ENDWHILE
12. RETURN $\{X(i)\}$ 
Remark 3 At a first look to steps 5 and 6 of Algorithm 1, the reader might think that the simulation of an infinite number of exponential variables is necessary in order to perform the algorithm. Actually, it is sufficient to simulate a finite number of finite valued random variables, see Knuth and Yao (1976) and also Section 10 of Galves et al. (2008).

\section{Proof of Theorem 1}

This section is devoted to the proof of Theorem 1, For the convenience of the reader, we start by recalling the following theorem of Galves et al. (2008).

Theorem 3 Under the requirement (4.34),

$$
\sup _{i \in \mathbb{Z}^{d}} \sum_{k \geq 1}\left|B_{i}(k)\right| \lambda_{i}(k)<1
$$

the Gibbs measure $\mu$ is the unique invariant probability measure of the process $\left(\sigma_{t}\right)_{t}$. The output $X(i)$ obtained using successively Algorithms 1 and 2 given in Section 4 is a perfect sampling of the Gibbs measure $\mu$. Finally, the estimate (4.35) holds :

$$
P\left(T_{S T O P}^{(i)}>t\right) \leq e^{-\gamma t}
$$

The same result holds true also for $X^{[L]}$ obtained using successively Algorithms 1 and 2 but now with restriction that only ranges $k \leq L$ are considered.

Remark 4 In Galves et al. (2008) the above result was proved for the minimal decomposition of the spin flip rates. The same proof works for the decomposition of the spin flip rates $c_{i}(\sigma)$ considered in Section 4. Note that in contrary to the decomposition considered in Galves et al. (2008), the decomposition in Section 4 is explicit in terms of the interaction.

We first check that the assumption (2.6) of Theorem 11implies condition (4.34) of Theorem 3.

Lemma 1 Under the conditions of Theorem 1, condition 4.34) is satisfied.

Proof For any $k \geq 0$, write for short

$$
S_{i}^{>k}=\sum_{B: i \in B, B \not \subset B_{i}(k)}\left|J_{B}\right| .
$$


For each $i \in \mathbb{Z}^{d}$ we have that

$$
\begin{aligned}
\sum_{k=1}^{\infty} \lambda_{i}(k)\left|B_{i}(k)\right| \leq & 2 d \lambda_{i}(1)+\sum_{k=2}^{\infty}\left|B_{i}(k)\right|\left[e^{-\beta S_{i}^{>k}}-e^{-\beta S_{i}^{>k-1}}\right] \\
= & 2 d e^{-\beta S_{i}^{>1}}\left(1-e^{-\beta S_{i}^{>0}} e^{-\beta \sum_{B: i \in B, B \subset B_{i}(1)}\left|J_{B}\right|}\right) \\
& +\sum_{k=2}^{\infty}\left|B_{i}(k)\right| e^{-\beta S_{i}^{>k}}\left[1-e^{-\beta \sum_{B: i \in B, B \subset B_{i}(k), B \not \subset B_{i}(k-1)}\left|J_{B}\right|}\right] \\
\leq & 2 d e^{-\beta S_{i}^{>1}}\left(1-e^{-\beta S_{i}^{>0}} e^{-\beta \sum_{B: i \in B, B \subset B_{i}(1)}\left|J_{B}\right|}\right) \\
& +\beta \sup _{i}\left(\sum_{k=2}^{\infty}\left|B_{i}(k)\right| \sum_{B: i \in B, B \subset B_{i}(k), B \not \subset B_{i}(k-1)}\left|J_{B}\right|\right) .
\end{aligned}
$$

Under the conditions of Theorem 1, this last expression is finite. Therefore condition (4.34) reads as

$$
\begin{aligned}
2 d \sup _{i}\left(e^{-\beta S_{i}^{>1}}\left(1-e^{-\beta S_{i}^{>0}} e^{-\beta \sum_{B: i \in B, B \subset B_{i}(1)}\left|J_{B}\right|}\right)\right) & \\
& +\beta \sup _{i}\left(\sum_{k=2}^{\infty}\left|B_{i}(k)\right| \sum_{B: i \in B, B \subset B_{i}(k), B \not \subset B_{i}(k-1)}\left|J_{B}\right|\right)<1 .
\end{aligned}
$$

Finally, let $\beta_{c}$ be the solution of

$$
\begin{aligned}
2 d \sup _{i}\left(e^{-\beta S_{i}^{>1}}\left(1-e^{-\beta S_{i}^{>0}} e^{-\beta \sum_{B: i \in B, B \subset B_{i}(1)}\left|J_{B}\right|}\right)\right) & \\
& +\beta \sup _{i}\left(\sum_{k=2}^{\infty}\left|B_{i}(k)\right| \sum_{B: i \in B, B \subset B_{i}(k), B \not \subset B_{i}(k-1)}\left|J_{B}\right|\right)=1 .
\end{aligned}
$$

This concludes the proof.

\section{Proof of Theorem 1 ,}

Proof of item 1. Theorem 3 combined with Lemma 1 proves that the output of the perfect sampling algorithm is indeed a sampling of $\mu$. Next we prove (2.7). Define

$$
L_{n}^{(i)}=\left|\mathbf{C}_{n}^{(i)}\right|
$$

the cardinal of the set $\mathbf{C}_{n}^{(i)}$ after $n$ steps of the algorithm. Let $\left(D_{n}^{(i)}\right)_{n \geq 0, i \in \mathbb{Z}^{d}}$ be i.i.d. random variables, independent of the process, taking values in $\{-1,0,1,2, \ldots\}$ such that for $k \geq 1$

$$
P\left(D_{n}^{(i)}=\left|B_{i}(k)\right|-1\right)=\lambda_{i}(k),
$$

and for $k=0$

$$
P\left(D_{n}^{(i)}=-1\right)=\lambda_{i}(0) .
$$

Note that under assumption (2.6), the condition (4.34) holds (see Lemma 1). Hence, $\sup _{i \in \mathbb{Z}^{d}} E\left(D_{1}^{(i)}\right) \leq-\gamma<0$. For any $i \in \mathbb{Z}^{d}$, let us call $I_{n}^{(i)}$ the index of the site whose 
Poisson clock rings at the $n$-th jump of the process $C^{(i)}$. Recall that $I_{n}^{(i)}$ are conditionally independent given the sequence $\left(\mathbf{C}_{n}^{(i)}\right)_{n}$ such that

$$
P\left(I_{n}^{(i)}=k \mid \mathbf{C}_{n-1}^{(i)}\right)=\frac{M_{k}}{\sum_{j \in \mathbf{C}_{n-1}^{(i)}} M_{j}} .
$$

Put

$$
S_{n}^{(i)}=\sum_{k=1}^{n} D_{k}^{I_{k}^{(i)}}
$$

Note that by construction, $S_{n}^{(i)}+n \gamma$ is a super-martingale. Then a very rough upper bound is

$$
L_{n}^{(i)} \leq 1+S_{n}^{(i)} \text { as long as } n \leq V_{S T O P}^{(i)},
$$

where $V_{S T O P}^{(i)}$ is defined as

$$
V_{S T O P}^{(i)}=\min \left\{k: S_{k}^{(i)}=-1\right\}
$$

By construction

$$
N_{S T O P}^{(i)} \leq V_{S T O P}^{(i)}
$$

Fix a truncation level $N$. Then by the stopping rule for super-martingales, we have that

$$
E\left(S_{V_{S T O P}^{(i)} \wedge N}^{(i)}\right)+\gamma E\left(V_{S T O P}^{(i)} \wedge N\right) \leq 0
$$

But notice that

$$
E\left(S_{V_{S T O P}^{(i)} \wedge N}^{(i)}\right)=-1 \cdot P\left(V_{S T O P}^{(i)} \leq N\right)+E\left(S_{N}^{(i)} ; V_{S T O P}^{(i)}>N\right)
$$

On $\left\{V_{S T O P}^{(i)}>N\right\}, S_{N}^{(i)} \geq 0$, hence we have that $E\left(S_{V_{S T O P}^{(i)} \wedge N}^{(i)}\right) \geq-P\left(V_{S T O P}^{(i)} \leq N\right)$. We conclude that

$$
E\left(V_{S T O P}^{(i)} \wedge N\right) \leq \frac{1}{\gamma} P\left(V_{S T O P}^{(i)} \leq N\right)
$$

Now, letting $N \rightarrow \infty$, we get

$$
E\left(V_{S T O P}^{(i)}\right) \leq \frac{1}{\gamma}
$$

and therefore

$$
E\left(N_{S T O P}^{(i)}\right) \leq \frac{1}{\gamma}
$$

Proof of item 2. We have to show that the coupled perfect sampling algorithm $G$ defined in Section 4 achieves the bound (2.8). For that sake we introduce the following stopping times. For any site $j \in \mathbb{Z}^{d}$ and any $t \geq 0$, let

$$
T_{1}^{(j, t)}=\inf \left\{T_{n}^{(j, 0)}>t\right\}-t
$$

be the first jump of the Poisson point process $\left(T_{n}^{(j, 0)}\right)_{n}$ shifted by time $t$. 
Let us call $T_{L}^{(i)}$ the first time that a range of order $k>L$ has been chosen. $T_{L}^{(i)}$ is given by

$$
T_{L}^{(i)}=\inf \left\{t>0: \sum_{j \in C_{t}^{(i)}} \sum_{k>L} N^{(j, k)}\left(\left[t, t+T_{1}^{(j, t)}[) \geq 1\right\} .\right.\right.
$$

Recall that in order to construct $\mu$ and $\mu^{[L]}$, we use the same Poisson point processes for all ranges $k \leq L$. Thus we have that

$$
P\left(X(i) \neq X^{[L]}(i)\right) \leq P\left(T_{L}^{(i)} \leq T_{S T O P}^{(i)}\right)
$$

By Lemma 2 given below we conclude the proof of Theorem 1 .

\section{Lemma 2}

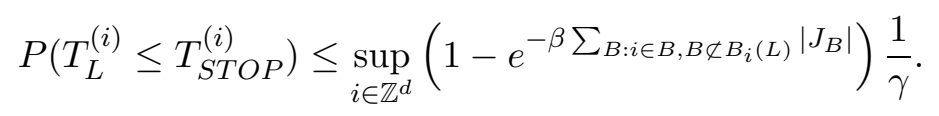

Proof Put

$$
\alpha_{i}(k)=\sum_{\ell \leq k} \lambda_{i}(\ell)
$$

Given the structure of the interaction, we have for $k \geq 2, \alpha_{i}(k)=e^{-\beta \sum_{B: i \in B, B \not \subset B_{i}(k)}\left|J_{B}\right|}$ and thus $1-\alpha_{i}(L) \leq \sup _{i \in \mathbb{Z}^{d}}\left(1-e^{-\beta \sum_{B: i \in B, B \not \subset B_{i}(L)}\left|J_{B}\right|}\right)$. Call this last quantity

$$
\delta(L)=\sup _{i \in \mathbb{Z}^{d}}\left(1-e^{-\beta \sum_{B: i \in B, B \not \subset B}(L)\left|J_{B}\right|}\right) .
$$

Then we have

$$
\begin{aligned}
& P\left(T_{L}^{(i)} \leq T_{S T O P}^{(i)}\right) \\
& \left.\left.\leq \sum_{n \geq 1} E\left(P\left(\left\{N^{(j, k)}(] \tilde{T}_{n-1}^{(i)}, \tilde{T}_{n}^{(i)}\right]\right)=1 \text { for } j \in \mathbf{C}_{n-1}^{(i)}, k>L\right\} \mid \mathbf{C}_{n-1}^{(i)}, N_{S T O P}^{(i)}>n-1\right)\right) \\
& =\sum_{n \geq 1} E\left(\sum_{j \in \mathbf{C}_{n-1}^{(i)}} \frac{M_{j}\left(1-\alpha_{j}(L)\right)}{\sum_{k \in \mathbf{C}_{n-1}^{(i)}} M_{k}} 1_{\left\{N_{S T O P}^{(i)}>n-1\right\}}\right) \\
& \leq \sum_{n \geq 1} E\left(\sum_{j \in \mathbf{C}_{n-1}^{(i)}} \frac{M_{j} \delta(L)}{\sum_{k \in \mathbf{C}_{n-1}^{(i)}} M_{k}} 1_{\left\{N_{S T O P}^{(i)}>n-1\right\}}\right) \\
& =\delta(L) E\left(N_{S T O P}^{(i)}\right) .
\end{aligned}
$$

In the above calculus we used that given $\mathbf{C}_{n-1}^{(i)}, \tilde{T}_{n}^{(i)}$ defined in (4.32) is a jump of $N^{(j, k)}$ with probability

$$
\frac{M_{j} \lambda_{j}(k)}{\sum_{k \in \mathbf{C}_{n-1}^{(i)}} M_{k}} \text {. }
$$

Summing over all possibilities $k>L$ gives the term $\left(M_{j}\left(1-\alpha_{j}(L)\right)\right) /\left(\sum_{k \in \mathbf{C}_{n-1}^{(i)}} M_{k}\right)$. By (4.36) we get the result.

Proof of Proposition 3 (4.35) is the statement of Theorem 3, (4.36) has been proved above in the proof of (2.7). 


\section{Final discussion}

It is worth to note that Theorem 11immediately provides an upper bound for the $\bar{d}$-distance of $\mu$ and $\mu^{[L]}$. The $\bar{d}$-distance is defined as follows.

Definition 5 Given two probability measures $\mu$ and $\nu$ on $S$, a coupling between $\mu$ and $\nu$ is a probability measure on $S \times S$ having as first and second marginals $\mu$ and $\nu$, respectively. The set of all couplings between $\mu$ and $\nu$ will be denoted $\mathcal{M}(\mu, \nu)$.

In the next definition, the elements of the product space $S \times S$ will be denoted by $\left(\left(\sigma_{1}(i), \sigma_{2}(i)\right), i \in \mathbb{Z}^{d}\right)$.

Definition 6 The distance $\bar{d}$ between two probability measures $\nu_{1}$ and $\nu_{2}$ on $S$ is defined as

$$
\bar{d}\left(\nu_{1}, \nu_{2}\right)=\inf _{Q \in \mathcal{M}\left(\nu_{1}, \nu_{2}\right)}\left\{\sup _{i \in \mathbb{Z}^{d}} Q\left(\sigma_{1}(i) \neq \sigma_{2}(i)\right)\right\} .
$$

This definition naturally extends Ornstein's $\bar{d}$-distance to the space of non-homogeneous random fields. As a corollary of Theorem 1 we obtain

Theorem 4 Under the assumptions of Theorem 11 we have

$$
\bar{d}\left(\mu, \mu^{[L]}\right) \leq C \sup _{i \in \mathbb{Z}^{d}}\left(1-e^{-\beta \sum_{B: i \in B, B \not \subset B_{i}(L)}\left|J_{B}\right|}\right)
$$

where $C$ is the same constant as in (2.8).

Upper bounds on the $\bar{d}$-distance can as well be obtained by using a contraction argument. This approach was introduced in Dobrushin (1968-1970) to prove uniqueness of the infinite volume Gibbs measure. Applied to our context, it implies the existence of a good coupling through compactness arguments.

For a nice and self contained presentation of this method, we refer the reader to Presutti (2009), see also Chapter 8 of Georgii. Applying Dobrushin's method, we obtain the following upper bound on Ornstein's $\bar{d}$-distance.

Theorem 5 Assume that

$$
\beta \sup _{i} \sum_{B: i \in B}\left|J_{B}\right|=r<1
$$

Then there exist unique infinite volume Gibbs measures $\mu$ and $\mu^{[L]}$ and they satisfy

$$
\bar{d}\left(\mu, \mu^{[L]}\right) \leq \frac{\beta}{1-r}\left(\sup _{i \in \mathbb{Z}^{d}} \sum_{B: i \in B, B \not \subset B_{i}(L)}\left|J_{B}\right|\right) .
$$

Proof The proof of Theorem 5 can be achieved following the ideas in Dobrushin (19681970), exposed in Chapter 3 of Presutti (2009). 
Observe that condition (6.46) is weaker than the one requested in Theorem[1. In particular, this condition is too weak to guarantee that our perfect simulation procedure of $\mu$ and $\mu^{[L]}$ stops after a finite number of steps. As a counterpart of the less restrictive assumption (6.46), the upper bound (6.47) is not as good as the upper bound (6.45).

However, from our point of view, the important fact is that the contraction method of Dobrushin is not constructive and does not provide an explicit sampling procedure for both measures. To provide an explicit coupled perfect simulation procedure for the measures is precisely the goal of the present paper.

Further features of high temperature region can be deduced by our constructive approach. We discuss as an example the decay of correlations between localized observables as the regions of localization are moved away from each other.

This decay of correlations can be described in terms of an associated random walk that we introduce now. Suppose that $\mu$ is translational invariant. In this case, the sequence $\left(\lambda_{i}(k)\right)_{k}$ does not depend on $i$, and we shall write $\lambda(k)=\lambda_{i}(k)$, for any $k \geq 0$. Let $\xi_{1}, \xi_{2}, \ldots$ be i.i.d. random variables with common distribution

$$
P\left(\xi_{1}=-1\right)=\lambda(0), \quad P\left(\xi_{1}=\left|B_{0}(k)\right|-1\right)=\lambda(k), \quad k \geq 1 .
$$

Put $\varphi(\lambda)=E\left(e^{\lambda \xi_{1}}\right)$ and let

$$
\rho=\sup \{\lambda>0: \varphi(\lambda) \leq 1\} .
$$

Finally, consider the following left-continuous random walk $S_{n}=\xi_{1}+\ldots+\xi_{n}$, for $n \geq 0$. Note that $S_{n}$ is an upper bound on the total number of sites whose spins affect the spin at site $i$ under equilibrium, after $n$ steps of the algorithm. Under condition (4.34), $E\left(\xi_{1}\right)=-\gamma<0$, hence $S_{n}$ drifts to $-\infty$. This implies that $M=\sup _{n} S_{n}$, the maximum of the random walk, is finite almost surely.

The following theorem is an immediate consequence of our construction and of results of Korshunov (1997) concerning the maximum of a random walk. In order to state the theorem, we have to recall the notion of locally power functions.

Definition 7 A function $f: \mathbb{R}_{+} \rightarrow \mathbb{R}_{+}$is called locally power if for every fixed $t \geq 0$, $f(x+t) \sim f(x)$ as $x \rightarrow \infty$, i.e. $\lim _{x \rightarrow \infty} \frac{f(x+t)}{f(x)}=1$.

Theorem 6 Take two cylinder functions $f$ and $g$ having support $\Delta_{f} \Subset \mathbb{Z}^{d}$ and $\Delta_{g} \Subset \mathbb{Z}^{d}$. Assume the distance $d\left(\Delta_{f}, \Delta_{g}\right)=R$. Under the assumption (4.34) we have

$$
|\mu(f g)-\mu(f) \mu(g)| \leq 2 c_{f} c_{g}\left(\left|\Delta_{f}\right|+\left|\Delta_{g}\right|\right) P(M \geq R / 2),
$$

where

$$
c_{f}=\sup _{i \in \Delta} \sup _{\sigma \in S}\left|f(\sigma)-f\left(\sigma^{i}\right)\right|
$$

and $c_{g}$ is similarly defined. Concerning the decay of $P(M \geq R / 2)$, the following holds.

1. Suppose that $\rho=0$ and that $P\left(\xi_{1}+\xi_{2} \geq n\right) \sim 2 P\left(\xi_{1} \geq n\right)$ as $n \rightarrow \infty$. Then as $n \rightarrow \infty$,

$$
P\left(M \geq\left|B_{0}(n)\right|\right) \sim \frac{1}{\gamma}\left[\sum_{k=n+1}^{\infty} \lambda(k)\left|B_{0}(k)\right|-\left|B_{0}(n)\right|\left(\sum_{k>n+1} \lambda(k)\right)\right]
$$

where $\gamma$ is given in (4.37). 
2. Suppose that $\rho>0, \varphi(\rho)<1$, that $P\left(\xi_{1}+\xi_{2} \geq n\right) \sim c P\left(\xi_{1} \geq n\right)$ as $n \rightarrow \infty$ for some constant $c$ and suppose that $e^{\rho n} P\left(\xi_{1} \geq n\right)$ is locally power. Then as $n \rightarrow \infty$

$$
P\left(M \geq\left|B_{0}(n)\right|\right) \sim \frac{\rho E\left(e^{\rho M}\right)}{1-\varphi(\rho)} \sum_{k=n+1}^{\infty} \lambda(k) .
$$

3. Suppose that $\rho>0, \varphi(\rho)=1$ and $\varphi^{\prime}(\rho)<+\infty$. Then

$$
P\left(M \geq\left|B_{0}(n)\right|\right) \sim c e^{-\rho n}
$$

as $n \rightarrow \infty$, where $c$ is some positive constant.

Remark 5 Using the same type of upper bounds as in the proof of Lemma 1, note that the expression

$$
\sum_{k=n+1}^{\infty} \lambda(k)\left|B_{0}(k)\right|
$$

that defines the decay of correlations can be upper bounded by

$$
\beta \sum_{k=n+1}^{\infty}\left(\sum_{B: 0 \in B, B \subset B_{0}(k), B \not \subset B_{0}(k-1)}\left|J_{B}\right|\right)\left|B_{0}(k)\right|,
$$

which yields the tail of the converging series of condition (2.6).

Proof We start by proving (6.48). It is sufficient to prove it for functions $f$ and $g$ having support $\Delta_{f}=\{i\}$ and $\Delta_{g}=\{j\}, R=\|i-j\|>0$. For any site $k \in \mathbb{Z}^{d}$, let

$$
\mathbf{C}^{(k)}=\bigcup_{n \geq 0} \mathbf{C}_{\mathbf{n}}^{(\mathbf{k})}
$$

be the total number of sites whose spins affect the spin of site $k$ under equilibrium (recall (4.33)). Then it is evident that

$$
|\mu(f g)-\mu(f) \mu(g)| \leq c_{f} c_{g}\left(\left|\Delta_{f}\right|+\left|\Delta_{g}\right|\right) P\left(\mathbf{C}^{(i)} \cap \mathbf{C}^{(j)} \neq \emptyset\right) .
$$

Then the same comparison argument that leads to (5.42) yields

$$
P\left(\mathbf{C}^{(i)} \cap \mathbf{C}^{(j)} \neq \emptyset\right) \leq P\left(M+M^{\prime} \geq R\right) \leq 2 P(M \geq R / 2) .
$$

This concludes the proof of (6.48).

Item 1 is a consequence of Theorem 1 of Korshunov (1997), see [10]. First notice that $\bar{F}(t)=P\left(\xi_{1}>t\right)$ is constant on intervals $\left[\left|B_{0}(n)\right|-1,\left|B_{0}(n+1)\right|-1[\right.$. Then evidently $\bar{F}\left(\left|B_{0}(n)\right|-1\right)=1-\alpha(n)$, where $\alpha_{n}=\lambda_{0}+\ldots+\lambda_{n}$. Therefore,

$$
\begin{aligned}
\int_{\left|B_{0}(n)\right|}^{\infty} \bar{F}(t) d t & =\left(\sum_{k=n}^{\infty}(1-\alpha(k))\left[\left|B_{0}(k+1)\right|-\left|B_{0}(k)\right|\right]\right)-(1-\alpha(n)) \\
& =\left(\sum_{k=n+1}^{\infty} \lambda(k)\left|B_{0}(k)\right|\right)-(1-\alpha(n))\left[\left|B_{0}(n)\right|+1\right] \\
& =\left(\sum_{k=n+1}^{\infty} \lambda(k)\left|B_{0}(k)\right|\right)-\left[\left|B_{0}(n)\right|+1\right]\left(\sum_{k>n} \lambda(k)\right) .
\end{aligned}
$$


This integral $\int_{\left|B_{0}(n)\right|}^{\infty} \bar{F}(t) d t$ is the quantity that determines the asymptotic behavior of $P\left(M \geq\left|B_{0}(n)\right|\right)$ as it is shown in Theorem 1 of Korshunov (1997). Items 2 and 3 are a consequence of Theorem 2 and Theorem 3 (ii) of Korshunov (1997).

\section{References}

[1] Dobrushin, R. L., The description of a random field by means of conditional probabilities and conditions of its regularity. Theor. Prob. Appl. 13, 197-224, (1968).

[2] Dobrushin, R. L., Gibbsian random fields for lattice systems with pairwise interactions. Funct. Anal. Appl. 2, 292-301, (1968).

[3] Dobrushin, R. L., The problem of uniqueness of Gibbs random field and the problem of phase transition. Funct. Anal. Appl. 2, 302-312, (1968).

[4] Dobrushin, R. L., Prescribing a system of random variables by conditional distributions. Theor. Prob. Appl. 302-312, (1970).

[5] Ferrari, P.A., Ergodicity for spin systems with stirrings. Ann. Probab. 18, No.4, 15231538 (1990).

[6] Ferrari, P.A.; Maass, A; Martínez, S; Ney, P., Cesàro mean distribution of group automata starting from measures with summable decay. Ergodic Theory Dynam. Systems 20, no. 6, 1657-1670 (2000).

[7] Galves, A.; Garcia, N. L.; Löcherbach, E., Perfect simulation and finitary coding for multicolor systems with interactions of infinite range. Preprint, arxiv.org/abs/0809.3494, (2008).

[8] Georgii, H.O., Gibbs measures and phase transitions. (1988), de Gruyter, Berlin.

[9] Knuth, D. E.; Yao, A. C., The complexity of nonuniform random number generation. Algorithms and complexity (Proc. Symp., Carnegie-Mellon Univ., Pittsburg, Pa. 1976), 375-428. Academic Press, New York.

[10] Korshunov, D., On distribution tail of the maximum of a random walk. Stoch. Process. Their Appl. 72, No.1, 97-103 (1997).

[11] Liggett, T., Interacting particles systems. (1985), Springer, Berlin.

[12] Presutti, E. Scaling Limits in Statistical Mechanics and Microstructures in Continuum Mechanics. Springer Berlin Heidelberg, Series: Theoretical and Mathematical Physics, 2009 
Antonio Galves

Instituto de Matemática e Estatística

Universidade de São Paulo

Caixa Postal 66281

05315-970 São Paulo, Brasil

e-mail: galves@usp.br

Eva Löcherbach

LAMA

Université Paris 12 Val de Marne

61 avenue du général de Gaulle

94000 CRETEIL CEDEX, France

email: locherbach@univ-paris12.fr

Enza Orlandi

Dipartimento di Matematica

Università di Roma Tre

L.go S.Murialdo 1, 00146 Roma, Italy.

email: orlandi@mat.uniroma3.it 